CMU-HEP90-13

FNAL-PUB-90/147-A

July, 1990

\title{
False-Vacuum Decay in Generalized Extended Inflation
}

\author{
Richard Holman, ${ }^{1}$ Edward W. Kolb, ${ }^{2}$ Sharon L. Vadas, ${ }^{3}$ Yun Wang, \\ ${ }^{1}$ Physics Department, Carnegie Mellon University, Pittsburgh PA 15219 \\ ${ }^{2}$ NASA/Fermilab Astrophysics Center \\ Fermi National Accelerator Laboratory, Batavia, IL 60510 \\ and \\ Department of Astronomy and Astrophysics and Enrico Fermi Institute \\ The University of Chicago, Chicago, IL 60637 \\ ${ }^{3}$ Physics Department, The University of Chicago, $\Pi 60697$
}

\begin{abstract}
We study false-vacuum decay in context of generalized extended inflationary theories, and compute the bubble nucleation rates for these theories in the limit of $G_{N} \rightarrow 0$. We find that the time dependence of the nucleation rate can be exponentially strong through the time dependence of the Jordan-BransDicke field. This can have a pronounced effect on whether extended inflation can be successfully implemented.
\end{abstract}

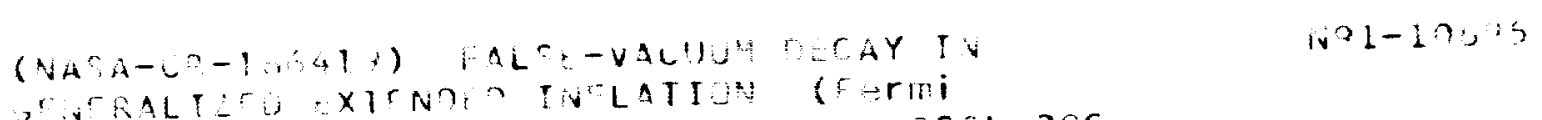

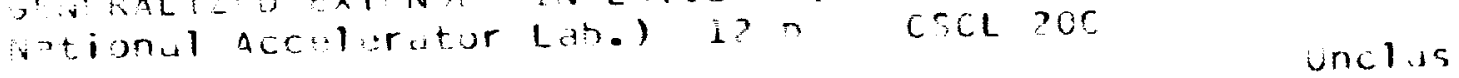

$$
\begin{aligned}
& \text { r.j/70 0 0 } 19 ? 2
\end{aligned}
$$


Extended inflation [1], one of the more interesting variants of a class of inflationary models, may be the key to solving the various problems faced by the standard cosmology. Extended inflation has revived the basic idea of old inflation [2], namely that inflation is induced by a field configuration trapped in a metastable state from which it exits via nucleation of bubbles of true vacuum. The original framework for extended inflation was the Jordan-Brans-Dicke theory [3] and involved two scalar fields, the inflaton and the Jordan-Brans-Dicke field. La and Steinhardt showed that in such a theory the expansion of the Universe is power law (rather than exponential) while dominated by the inflaton false-vacuum energy. This implies that the true vacuum phase could percolate, and thus the graceful exit problem of old inflation [4] could be evaded [1].

Unfortunately, in the La-Steinhardt model the requirements that the bubble-wall energy be thermalized before any cosmologically sensitive times (such as nucleosynthesis or recombination) and that a global Robertson-Walker frame be reestablished in the bubble cluster, resulted in a physically unacceptable upper bound on the Brans-Dicke parameter $\omega$ [5]. Weinberg showed that this upper bound is of order 20 [5], while the experimental lower bound is $\omega>500$ [6]! Thus, if one wishes to make use of extended inflation, ways of avoiding this unpleasant problem must be found.

More sophisticated models have been considered, such as those which fix the present value of the Jordan-Brans-Dicke scalar field [7], as well as theories in which the inflaton couples to gravity with a different strength than normal matter [8]. Extended inflation models within the framework of Kaluza-Klein theories [9] have also been examined [10], as have the effects of higher order curvature terms on extended inflation [11], and the effect of a generalization of the coupling of the Jordan-Brans-Dicke scalar field to the curvature scalar [12].

The crux of the graceful exit problem concerns the so-called percolation parameter $\epsilon \equiv \lambda / H^{4}$, where $\lambda$ is the bubble nucleation rate per physical three-volume and $H$ is 
the Hubble parameter during inflation. The quantity $\epsilon$ is a measure of the number of true-vacuum bubbles nucleated within a Hubble volume $H^{-3}$ in a Hubble time $H^{-1}$. The graceful exit problem in old inflation is essentially the following: In order for enough inflation to be achieved we must have $\epsilon \lesssim 4 \times 10^{-3}$ [4], while in order for the true vacuum phase to percolate, we must demand that $\epsilon \gtrsim \epsilon_{C R}$, with $10^{-6} \lesssim \epsilon_{C R} \lesssim 0.24$. Now, both $\lambda$ and $H$ are constant during inflation in the old inflationary scenario. Since $\lambda$ is exponentially small in general, extreme fine-tuning would be required in order to satisfy both constraints (if it could be done at all!).

In the model of $\mathrm{La}$ and Steinhardt, the fact that the super-luminal expansion is a power-law makes $H(t)$ time dependent, while $\lambda$ remains time-independent (despite the time evolution of the Jordan-Brans-Dicke (JBD) field $\Phi[13]$ ). This in turn allows $\epsilon$ to increase in time so that it can start small enough to allow for sufficient inflation, and then grow to be large enough that $\epsilon>\epsilon_{C R}$ so that percolation occurs.

Previously [13], we showed that the original model of extended inflation with a variable gravitational coupling can be recast by a conformal transformation as a theory with a "constant" Newton's constant, but at the cost of introducing a complicated coupling between the inflaton field and the Jordan-Brans-Dicke scalar field. Although the technique of the conformal transformation has been employed before in order to analyze inflationary models with varying gravitational constant [14], it had not been appreciated previously that the nucleation rate will have a different time dependence in the new conformal frame than it had in the original frame.

The fact that extended inflation can be viewed in a conformal frame where gravity is "normal" suggests that the physical justification for extended inflation may arise from modification of the scalar sector of particle physics rather than modification of the fundamental gravitational interaction. ${ }^{1}$ In fact, the possibility that the evolution of a

\footnotetext{
${ }^{1}$ Of course in many cases it is possible to perform a conformal transformation and express the theory
} 
scalar field can induce a first-order phase transition in another scalar field was realized by Kofman and Linde [15]. In their model the effective mass of the field involved in the phase transition was slowly changed by the evolution of some other field.

For the moment, consider a theory in the conformal frame with a constant coefficient of the Ricci scalar. All extended inflation models to date can be expressed in terms of two degrees of freedom; an inflaton degree of freedom trapped in the metastable vacuum, and a JBD degree of freedom evolving in time (slowly in most cases). In the model of Kofman and Linde, the JBD degree of freedom only couples to the inflaton mass term, while in the model of La and Steinhardt, the JBD degree of freedom couples to both the potential and kinetic terms for the inflaton in a prescribed way. In general the coupling of the JBD degree of freedom to the inflaton field is model dependent $[8,10]$. Since the nucleation rate $\lambda$ enters into the crucial parameter $\epsilon$, it is important to be able to calculate the influence of the JBD field upon the decay rate of the false vacuum. In this paper, we consider the most general couplings of the JBD field to the inflaton and calculate the bubble nucleation rate per unit three-volume. We find that the generic situation is that $\lambda$ acquires an exponential dependence on the JBD field. This then allows for many more possibilities in terms of the behaviour of $\epsilon$. For example, even if $H(t)$ increases in time (unlike the case in the original extended inflation scenario), $\epsilon(t)=\lambda(t) / H^{4}(t)$ can still increase $[10]$ in time.

Let us start by considering theories of a JBD field $\Phi$ coupled to an inflaton field $\sigma$ via the following generic action:

$$
\begin{aligned}
S=\int d^{4} x \sqrt{-g}\left[-\Phi R+\omega g^{\mu \nu} \frac{\partial_{\mu} \Phi \partial_{\nu} \Phi}{\Phi}\right. \\
\left.+F(\Phi) \frac{1}{2} g^{\mu \nu} \partial_{\mu} \sigma \partial_{\nu} \sigma-G(\Phi) V(\sigma)\right] .
\end{aligned}
$$

Here we have written the action in the Jondan Conformal Frame, where Newton's conin a form that resembles Jordan-Brans-Dicke, and the question of whether it is the gravity sector or the scalar sector that one is modifying is a matter of taste. 
stant $G_{N}$ is replaced by the JBD field $\Phi$ in the curvature term. The field $\sigma$ is the inflaton field and its potential has a metastable (false-vacuum) minimum at $\sigma=\sigma_{F V}$.

The simplest coupling functions can be of the form

$$
F(\Phi)=\left(16 \pi G_{N} \Phi\right)^{n}, \quad G(\Phi)=\left(16 \pi G_{N} \Phi\right)^{m}
$$

To ensure that our theory reduces to general relativity in the appropriate limit, we require that for $\Phi=\Phi_{0}=1 / 16 \pi G_{N}, F\left(\Phi_{0}\right)=G\left(\Phi_{0}\right)=1$.

We note that the standard formalisms (the Euclidean bounce method) for performing these calculations developed by Coleman and Callan [16] (and generalized by Coleman and DeLuccia to include classical gravity [17]) are not immediately applicable to the problem at hand. In extended inflation models, the time evolution of JBD field $\Phi$ and its non-trivial couplings to the inflaton cause the false vacuum to "roll" during the bounce, thus presenting a problem more complicated than in standard false-vacuum tunneling. As far as extended inflation theories are concerned, one cannot exagerate the importance of understanding the mechanism of false-vacuum tunneling under such circumstances. It is a very difficult task. Some work has been done toward this goal by Accetta and Romanelli [18]. It may, however, be more useful to attempt to understand the tunnelling problem in this system via a Hamiltonian formalism [19]. In this letter, however, we want to elaborate on the method developed by us [13] which allows us to systematically "freeze out" gravitational effects in the bounce, thus enabling us to arrive at approximate expressions for the nucleation rate which reflect the time evolution of the JBD field $\Phi$.

To implement our approximation, we go to the Einstein Conformal Frame. The reason comes from the observation that in the Jordan conformal frame action [i.e., Eq. (1)], the second term is not the complete kinetic term for $\Phi$, since an integration by parts of the first term will make a contribution to the $\Phi$ kinetic term. Therefore, for semi-classical calculations involving the JBD field, it is more appropriate (and often easier) to use 
the Einstein Conformal Frame, where the gravitational couplings are the standard ones. Then we may transform back to the Jordan frame if we choose. Thus we perform the following conformal transformation

$$
\begin{aligned}
g_{\mu \nu} & =\Omega^{2} \bar{g}_{\mu \nu} \\
\sqrt{-g} & =\Omega^{4} \sqrt{-\bar{g}} \\
R & =\Omega^{-2} \bar{R}-6 \Omega^{-3} \bar{\square} \Omega
\end{aligned}
$$

where the overbars denote quantities in the Einstein conformal frame, and $\Omega^{-2}=16 \pi G_{N} \Phi$. We define a new field $\psi$ via the relation

$$
\psi \equiv \psi_{0} \ln \left(16 \pi G_{N} \Phi\right)
$$

with $\psi_{0}^{2} \equiv(3+2 \omega) / 16 \pi G_{N}$. With these definitions, the action of Eq. (1) expressed in the Einstein frame becomes

$$
\begin{aligned}
\bar{S}=\int d^{4} x \sqrt{-\bar{g}}\left[-\frac{\bar{R}}{16 \pi G_{N}}+\frac{1}{2} \bar{g}^{\mu \nu} \partial_{\mu} \psi \partial_{\nu} \psi\right. \\
\left.\quad+f\left(\psi / \psi_{0}\right) \frac{1}{2} \bar{g}^{\mu \nu} \partial_{\mu} \sigma \partial_{\nu} \sigma-g\left(\psi / \psi_{0}\right) V(\sigma)\right] .
\end{aligned}
$$

where

$$
f\left(\psi / \psi_{0}\right) \equiv \exp \left(-\psi / \psi_{0}\right) F(\Phi), \quad g\left(\psi / \psi_{0}\right) \equiv \exp \left(-2 \psi / \psi_{0}\right) G(\Phi)
$$

and $\Phi=\Phi\left(\psi / \psi_{0}\right)$ is understood. For the simple couplings of Eq. (2), $f(\psi)=\exp [(n-$ 1) $\left.\psi / \psi_{0}\right]$ and $g(\psi)=\exp \left[(m-1) \psi / \psi_{0}\right]$.

The action written as above in the Einstein frame reveals that if we want to freeze out gravitational effects, we must also freeze out the evolution of the $\psi$ field during the bounce. This is due to the fact that we are taking the $G_{N} \rightarrow 0$ limit and $\psi_{0} \propto G_{N}^{-1 / 2}$, thus the second term has the same $G_{N}$ dependence as the first term. Treating $\psi$ as constant also implies that $\Phi$ must be taken to be constant. This may be alarming since 
we have to use explicitly the fact that both the scale factor and $\Phi$ are time dependent to make our discussion relevant for inflation. However, we are saved by the observation that the (imaginary-time) bounce configuration used in computing the tunneling action is distinct from the (real-time) background metric and JBD field configuration governing the evolution of the Universe. Thus the latter can remain time dependent while we freeze out the time evolution in $\psi$ during the tunneling process [13].

Corrections to this approximation can also be considered. We expect, using the results of bubble nucleation calculations in standard gravity as a guide, that our approximation will be reliable when the effective Planck mass induced by the JBD field is much greater than the mass scales associated with the $\sigma$ field. In theories where $\Phi$ increases with time, the approximation will work best at late times.

The approximation discussed above yields the following truncated action for the inflaton $\sigma$ in the Euclidean frame:

$$
\bar{S}_{E}=\int d^{4} x\left[f(\xi) \frac{1}{2} \partial^{\mu} \sigma \partial_{\mu} \sigma+g(\xi) V(\sigma)\right]
$$

where $\xi \equiv \exp \left(\psi / \psi_{0}\right)$. We remind the reader that this might very well be considered the starting point for extended inflation, without recourse to the Jordan-Brans-Dicke action. In this case one must still ignore the evolution of $\xi$ in calculating the bounce. Although this might very well be a good approximation, there is no systematic reason for doing so unless the $\xi$ field is of gravitational origin, in which case it may safely be ignored in the $G_{N} \rightarrow 0$ limit as discussed above.

To calculate the bubble nucleation rate (per unit physical three volume)

$$
\bar{\lambda}=A \exp (-B)
$$

we need to calculate the bounce action, $B$, and the prefactor, $A[16]$. 
If we rescale the coordinates to

$$
\widehat{x}^{\alpha}=\sqrt{\frac{g(\xi)}{f(\xi)}} x^{\alpha}
$$

we can rewrite the action of Eq. (7) as

$$
\bar{S}_{E}=\frac{f^{2}(\xi)}{g(\xi)} \int d^{\alpha} \bar{x}\left[\frac{1}{2} \hat{\partial}^{\mu} \sigma \widehat{\partial}_{\mu} \sigma+V(\sigma)\right]=\frac{f^{2}(\xi)}{g(\xi)} S_{0}
$$

where $S_{0}$ is the Euclidean action of the standard theory (i.e., the action of Eq. (5) with $\xi=1)$. Clearly, this implies that the bounce configuration $\sigma_{B}$ is related to the bounce of the theory containing $\widehat{\sigma}_{B}$ :

$$
\sigma_{B}(x)=\widehat{\sigma}_{B}(\sqrt{g(\xi) / f(\xi)} x) .
$$

The bounce action is

$$
B(\xi)=\frac{f^{2}(\xi)}{g(\xi)} B_{0} \quad\left[\xi=\exp \left(\psi / \psi_{0}\right)\right],
$$

where $B_{0}$ is the ( $\xi$-independent) bounce action calculated for the theory with $\xi=1$ $(\psi=0)$. The fact that the coupling of $\psi$ into the action of Eq. (7) can be factored out by means of coordinate rescaling is essential in enabling us to carry out our calculation.

The prefactor $A$ from Eq. (8) is given by [16]

$$
A=\left|\frac{\operatorname{det}^{\prime}\left[S_{E}^{\prime \prime}\left(\sigma_{B}\right)\right]}{\operatorname{det}\left[S_{E}^{\prime \prime}\left(\sigma_{F V}\right)\right]}\right|^{-1 / 2} \prod_{\mu}\left(\frac{C_{\mu}}{2 \pi}\right)^{1 / 2} .
$$

Here, $\sigma_{F V}$ is the false-vaccum configuration, $\sigma_{B}$ is the bounce solution, and det' indicates that the functional determinant is to be evaluated in the subspace orthogonal to the four translational zero modes. The $C_{\mu}$ are normalization factors of the zero modes of the operator $S_{E}^{\prime \prime}\left(\sigma_{B}\right)$.

Performing the functional variation of the Euclidean action yields

$$
\begin{aligned}
A_{D E T} & \equiv\left|\frac{\operatorname{det}^{\prime}\left[S_{E}^{\prime \prime}\left(\sigma_{B}\right)\right]}{\operatorname{det}\left[S_{E}^{\prime \prime}\left(\sigma_{F V}\right)\right]}\right|^{-1 / 2} \\
& =\left|\frac{\operatorname{det}^{\prime}\left[-f(\xi) \partial^{2}+g(\xi) V^{\prime \prime}\left(\sigma_{B}\right)\right]}{\operatorname{det}\left[-f(\xi) \partial^{2}+V^{\prime \prime}\left(\sigma_{F V}\right)\right]}\right|^{-1 / 2} .
\end{aligned}
$$


To determine the $\xi$ dependence of the above expression, we observe that if $\Psi_{\theta}(x)$ is the eigenfunction of the operator $-\hat{\partial}^{2}+V^{\prime \prime}(\hat{\sigma})$ with eigenvalue $\theta$, then

$$
\begin{aligned}
{\left[-f(\xi) \partial^{2}+g(\xi) V^{\prime \prime}(\sigma)\right] \Psi_{\theta}(\sqrt{g(\xi) / f(\xi)} x) } & =g(\xi)\left[-\widehat{\partial}^{2}+V^{\prime \prime}(\sigma)\right] \Psi_{\theta}(\widehat{x}) \\
& =g(\xi) \theta \Psi_{\theta}(\sqrt{g(\xi) / f(\xi)} x)
\end{aligned}
$$

i.e., $\Psi_{\theta}(\sqrt{g(\xi) / f(\xi)} x)$ is the eigenfunction of the operator $-f(\xi) \partial^{2}+g(\xi) V^{\prime \prime}\left(\sigma_{B}\right)$ with eigenvalue $g(\xi) \theta$. Since the primed determinant has four eigenvalues fewer than the unprimed one, we have

$$
A_{D E T}=\left\{[g(\xi)]^{-4}\right\}^{-1 / 2} \hat{A}_{D E T}=g^{2}(\xi) \hat{A}_{D E T}
$$

The $C_{\mu}$ are defined so that the properly normalized modes are $C_{\mu}^{-1 / 2} \partial_{\mu} \sigma_{B}(\mu=$ $1, \cdots, 4)$. Thus, $C_{\mu}=\int d^{4} x\left(\partial_{\mu} \sigma_{B}\right)^{2}$ (no sum over $\mu$ implied), and for an $O(4)$-symmetric bounce, the $C_{\mu}$ are all equal. The $\xi$ dependence of $C_{\mu}$ can easily be found:

$$
C_{\mu}=\int d^{4} x\left(\partial_{\mu} \sigma_{B}\right)^{2}=f(\xi) / g(\xi) \hat{C}_{\mu} .
$$

Hence, the nucleation rate in the Einstein frame is

$$
\bar{\lambda}(\bar{t})=f^{2}(\xi) \hat{A} \exp \left(-B_{0} f^{2}(\xi) / g(\xi)\right)
$$

Now we may find the nucleation rate in the Jordan frame. Recall that $\exp \left(\psi / \psi_{0}\right)=$ $16 \pi G_{N} \Phi$, and that the nucleation rate in the Jordan frame is related to that in the Einstein frame by

$$
\begin{aligned}
\lambda & \equiv \frac{d P}{d^{4} x \sqrt{-g}}=\frac{d P}{d^{4} x \sqrt{-\bar{g}}} \frac{\sqrt{-\bar{g}}}{\sqrt{-g}}=\Omega^{-4} \bar{\lambda} \\
& =\xi^{2} f^{2}(\xi) \hat{A} \exp \left(-B_{0} f^{2}(\xi) / g(\xi)\right) \\
& =\hat{A} F^{2}(\Phi) \exp \left\{-B_{0} F^{2}(\Phi) / G(\Phi)\right\}
\end{aligned}
$$

$\hat{A}$ and $B_{0}$ are $\Phi$ independent and depend only upon the inflaton potential. $B_{0}$ is dimensionless, while $\widehat{A}$ has mass dimension 4. 
For the simple power-law coupling functions in Eq. (2), we have

$$
\lambda=\widehat{A}\left(16 \pi G_{N} \Phi\right)^{2 n} \exp \left[-B_{0}\left(16 \pi G_{N} \Phi\right)^{2 n-m}\right]
$$

In the original extended inflation model $m=n=0$, and in the Jordan frame the nucleation rate is time independent, although it is time dependent in the Einstein frame (as discussed in [13]). However, in dimensionally reduced theories, the generic form has $m$ and $n$ different from zero [10]. We see from the above equation that if $2 n-m \neq 0$ the time dependence of the nucleation probability can be exponentially strong through the time dependence of $\Phi$ (or equivalently, $\psi$ ). If $2 n-m=0$ but $n \neq 0$, the nucleation probability is still time dependent in the Jordan frame, and time dependent in the Einstein frame if $n \neq 1$.

For arbitrary functions $F(\Phi)$ and $G(\Phi)$, we can expect much richer time dependence of the bubble nucleation rate.

To conclude, we have found that in generalized extended inflation theories, the bubble nucleation rate acquires an explicit time dependence, even in the limit of freezing out gravitational effects. The time dependence will be exponentially strong in the generic case. This remarkable feature of the theories encompassed by our model provides optimistic prospects for the success of percolation, since the time dependence of the percolation parameter $\epsilon$ is enriched through the time dependence of the nucleation probability.

\section{Acknowledgements}

The work of R.H. and Y.W. was supported in part by the Department of Energy grant \#DE-AC02-76ER3066, while the work of E.W.K and S.V. was supported in part by the Department of Energy and NASA (grant \#NAGW-1340). 


\section{References}

1. D. La and P. J. Steinhardt, Phys. Rev. Lett. 62 (1989) 376; Phys. Lett. 220B (1989) 375.

2. A. H. Guth, Phys. Rev. D 23 (1981) 347.

3. P. Jordan, Zeit. Phys. 157 (1959) 112; C. Brans and C. H. Dicke, Phys. Rev. 24 (1961) 925.

4. A. H. Guth and E. W. Weinberg, Nucl. Phys. B212 (1982) 321.

5. E. J. Weinberg, Phys. Rev. D 40 (1989) 3950; D. La, P. J. Steinhardt and E. Bertschinger, Phys. Lett. 231B (1989) 231.

6. R. D. Reasenberg, et al., Astrophys. J. 234 (1979) L219.

7. F. S. Accetta and J. J. Trester, Phys. Rev. D 39 (1989) 2854.

8. R. Holman, E. W. Kolb, and Y. Wang, Phys. Rev. Lett. 65 (1990) 17.

9. T. Kaluza, Preus. Acad. Wiss. K1 (1921) 966; O. Klein, Zeit. Phys. 37 (1926) 895; and Nature 118 (1926) 516; M. J. Duff, B. E. W. Nilsson, and C. N. Pope, Phys. Rep. 130 (1986) 1.

10. R. Holman, E. W. Kolb, S. Vadas, Y. Wang, Carnegie-Mellon University preprint CMU-HEP90-09, Fermilab preprint FNAL-PUB-90/99-A (1990) (submitted to Phys. Rev. D).

11. Y. Wang, Carnegie-Mellon University preprint CMU-HEP90-10 (1990) (to appear in Phys. Rev. D).

12. P. J. Steinhardt and F. Accetta, Phys. Rev. Lett. 64 (1990) 2740. 
13. R. Holman, E. W. Kolb, S. L. Vadas, Y. Wang, and E. J. Weinberg, Phys. Lett. 237B (1990) 37.

14. K. Maeda, Phys. Rev. D 39 (1989) 3159; D. S. Salopek, J. R. Bond, and J. M. Bardeen, Phys. Rev. D 40 (1989) 1753.

15. L. A. Kofman and A. D. Linde, Nucl. Phys. B282 (1987) 555; A. D. Linde, CERN preprint CERN-TH.5806/90 (1990).

16. S. Coleman, Phys. Rev. D 15 (1977) 2929; C. G. Callan and S. Coleman, Phys. Rev. D 16 (1977) 1762.

17. S. Coleman and F. De Luccia, Phys. Rev. D 21 (1980) 3305.

18. F. S. Accetta and P. Romanelli, Phys. Rev. D 41 (1990) 3024.

19. A. S. Goncharov and A. D. Linde, Sov. J. Part. Nucl. 17 (1986) 369; N. Deruelle, CERN preprint CERN.TH.5331/89 (1989); W. Fischler, D. Morgan, and J. Polchinski, Preprint UTTG-17-90 (1990). 
